\title{
QUANTUM GROUPS, INTEGRABLE STATISTICAL MODELS AND KNOT THEORY
}


This page is intentionally left blank 
Nankai Lectures on Mathematical Physics

\title{
QUANTUM GROUPS, \\ INTEGRABLE STATISTICAL MODELS \\ AND KNOT THEORY
}

Nankai Institute of Mathematics, China

11 - 13 June 1992

\author{
Editors \\ M. L. Ge \\ Nankai University \\ H. J. de Vega \\ Laboratoire de Physique Théorique \\ et Hautes Énergies \\ Paris
}




\section{Published by}

World Scientific Publishing Co. Pte. Ltd.

P O Box 128, Farrer Road, Singapore 9128

USA office: Suite 1B, 1060 Main Street, River Edge, NJ 07661

UK office: 73 Lynton Mead, Totteridge, London N20 8DH

\section{QUANTUM GROUPS, INTEGRABLE STATISTICAL MODELS AND KNOT THEORY}

Copyright 1993 by World Scientific Publishing Co. Pte. Ltd.

All rights reserved. This book, or parts thereof, may not be reproduced in any form or by any means, electronic or mechanical, including photocopying, recording or any information storage and retrieval system now known or to be invented, without written permission from the Publisher.

For photocopying of materials in this volume, please pay a copying fee through the Copyright Clearance Center, Inc., 27 Congress Street, Salem, MA 01970, USA.

ISBN 981-02-1474-X

Printed in Singapore by Stamford Press Pte Ltd 


\section{Preface}

The Yang-Baxter equations and their solutions are at the heart of some of the more important progress in mathematical physics and pure mathematics in the last years.

A rich variety of statistical lattice and quantum continuum two dimensional models follows from the Yang-Baxter equations. For these lattice integrable models the main physical magnitudes as free energy, excitation spectrum, critical exponents, one-point correlators, etc. can be exactly computed. In the $1+1$ dimensional integrable field theories associate to Yang-Baxter solutions, the mass spectrum, the S-matrix and form factors are explicitly computable. These models have a rich physical content and relevant applications in statistical and condensed matter physics. In pure mathematics, the discovery of the quantum groups was achieved investigating integrable two dimensional models. In addition, knot theory positively benefited from the application of Yang-Baxter solutions in the calculation of knot invariants.

The Fifth Nankai Workshop held at Nankai University from 11 to 13 June 1992 intended to cover some selected developments in this rapidly progressing domain where mathematical physics and pure mathematics meet in a deep and fruitful way.

It is a pleasure to thank all the speakers for their successful efforts in delivering interesting and stimulating lectures and for thier careful written contributions. We thank all the participants for their interest and discussions.

H. J. de Vega

Ge Mo-Lin 
This page is intentionally left blank 


\section{Table of Contents}

Preface

Lectures on Quantum Groups: Representations and Duality

V. K. Dobrev

Bethe Ansatz and Quantum Groups

H. J. de Vega

W-Geometries and Two-Dimensional Gravity

J.-L. Gervais

Spectral Problem in the Quantum Inverse Scattering Method.

The Goryachev-Chaplygin Top

I. V. Komarov

Vassiliev Invariants and the Jones Polynomial

L. H. Kauffman

Aspects of Yangian-Invariant Factorized S-matrices

N. J. Mackay

Beyond Supersymmetry and Quantum Symmetry. (An Introduction to Braided-Groups and Braided-Matrices)

S. Majid

Q-Deformation of the Conformal Algebra

Z. Popowicz

Infinite-Dimensional $R$ Matrix with Complete $\mathbb{Z}$ Symmetry .

Y. Shibukawa

On Representation Theories of Iwahori-Hecke Algebras $H q(W)$ at Roots $q$ of Unity. (In Particular, Explicit Formulas $H_{n_{\sqrt{1}}}\left(S_{n}\right)$ and $\left.H_{n-1 \sqrt{1}}\left(S_{n}\right)\right)$

H. Yamane 